\title{
Large-Area Nanoimprint Lithography and Applications
}

\author{
Hongbo Lan
}

Additional information is available at the end of the chapter

http://dx.doi.org/10.5772/intechopen.72860

\begin{abstract}
Large-area nanoimprint lithography (NIL) has been regarded as one of the most promising micro/nano-manufacturing technologies for mass production of large-area micro/ nanoscale patterns and complex 3D structures and high aspect ratio features with low cost, high throughput, and high resolution. That opens the door and paves the way for many commercial applications not previously conceptualized or economically feasible. Great progresses in large-area nanoimprint lithography have been achieved in recent years. This chapter mainly presents a comprehensive review of recent advances in largearea NIL processes. Some promising solutions of large-area NIL and emerging methods, which can implement mass production of micro-and nanostructures over large areas on various substrates or surfaces, are described in detail. Moreover, numerous industriallevel applications and innovative products based on large-area NIL are also demonstrated. Finally, prospects, challenges, and future directions for industrial scale largearea NIL are addressed. An infrastructure of large-area nanoimprint lithography is proposed. In addition, some recent progresses and research activities in large-area NIL suitable for high volume manufacturing environments from our Labs are also introduced. This chapter may provide a reference and direction for the further explorations and studies of large-area micro/nanopatterning technologies.
\end{abstract}

Keywords: large-area nanoimprint lithography, large-area micro/nanopatterning, full wafer NIL, roller-type NIL, roll-to-plate NIL, roll-to-roll NIL

\section{Introduction}

Mass producing nanostructure over large areas is critical to the commercial applications of nanotechnology. Large-area nanopatterning has demonstrated great potential which can significantly enhance the performance of many devices and create innovative products, such as LEDs, solar cells, hard disk drives, laser diodes, displays, sub-wavelength optical elements, antireflective glass with moth's eye structures, flexible electronics, OLED, etc. [1-7]. For example, the 
solar cells with submicro anti-reflective coating exhibited higher photocurrent and higher power conversion efficiency compared to those without nanostructures [8]. Nano-patterned sapphire substrates (NPSS) and photonic crystals (PhC) have been considered as the most effective approaches to improve the light output efficiency (internal quantum efficiency and external quantum efficiency) of LEDs and beam shaping [9, 10]. However, mass producing large-area nanostructures, particularly nanopatterning on various curved or non-flat surfaces or fragile substrates or flexible substrates, are particularly difficult using current patterning methods. Furthermore, a variety of existing micro/nano-manufacturing technologies such as optical lithography, electron beam lithography, focused ion beam lithography, interference lithography, etc., cannot cope with all the practical requirements of industrial scale applications with respect to high resolution, high throughput, low cost, large-area, especially patterning on non-flat and curved surface. It is now still a challenging issue to produce large-area micro/nanoscale structures with low cost, high throughput for existing micro/nano-manufacturing technologies [11].

Nanoimprint lithography (NIL) has now been regarded as a promising nanopatterning approach with low cost, high throughput, and high resolution, especially for fabricating the largearea micro/nanoscale patterns and complex 3D structures and high aspect ratio features. Due to these outstanding strengths and unique capabilities, it was accepted by International Technology Roadmap for Semiconductors (ITRS) in 2009 for 16 and $11 \mathrm{~nm}$ nodes, scheduled for industrial manufacturing in 2013. Toshiba has validated NIL for $22 \mathrm{~nm}$ and beyond. NIL has been listed as one of 10 emerging technologies that will strongly impact the world by MIT's Technology Review. The resolution potential has been demonstrated by the replication of 2.4-nm features. It is expected to play a critical role in the commercialization of nanostructure applications [12-14]. In particular, a variety of emerging large-area NIL processes (e.g. waferscale soft UV-NIL process, roll-to-plate NIL, roll-to-roll NIL process) provide ideal solutions and powerful tools for mass producing micro/nanostructures over large areas and continuous patterning at low cost and high yield rate for the industrial scale applications in compound semiconductor optoelectronics, wafer-level optical element, and nanophotonic devices, especially for LED patterning using full wafer NIL and anti-reflective films or coatings by roll-toroll nanoimprint process. That opens the door and paves the way for many commercial applications not previously conceptualized or economically feasible.

A large number of studies and efforts regarding large-area nanoimprint lithography have been carried out by both academia and industries. Great progresses in large-area nanoimprint lithography have been achieved in recent years. This chapter focuses on the significant progresses in large-area NIL processes and industrial applications. The rest of the chapter is organized as follows. Typical and emerging large-area NIL processes are discussed in detail in Section 4. Furthermore, Section 3 presents some industrial applications of large-area NIL. Prospects, challenges, and future directions for industrial scale large-area NIL are elaborated in Section 5. Finally, Section 6 summaries this chapter.

\section{Large-area NIL processes}

A variety of large-area NIL processes have been proposed and developed by both academia and industries. According to the types of used molds, type of imprint contact, and imprinting 
continuity, large-area NIL technology can be classified into two fundamental categories: the full wafer NIL and roller-type NIL. Roller-type NIL can be further divided into two subclasses: roll-to-plate (R2P) NIL and roll-to-roll (R2R) NIL. The R2R NIL process has shown a highly promising future to be implemented as a full-scale production process due to their high throughput and large-area patterning capability. The continuous roller-pressing process has been currently applied in many industrial fields [15-17].

\subsection{Full wafer NIL (plate-to-plate type NIL, batch press type)}

In full wafer NIL, a (flexible) flat mold/stamp is utilized to press into a resist layer on a rigid substrate, resulting in a full field contact. In order to pattern a whole wafer area without the high imprinting forces and demolding force, soft UV-NIL using a flexible (or soft) mold has been proven to be a very promising approach for producing large-area patterns up to waferlevel in the micrometer and nanometer scale. The process possesses the following outstanding strengths: (1) flexible mold using; (2) sequential imprinting; and (3) peel-off demolding. Substrate conformal imprint lithography (SCIL) developed by Philips Research and SUSS MicroTec, is a novel large-area wafer-scale nanoimprint method with nanoscale resolution. It bridges the gap between small rigid imprint stamp for best resolution and large-area soft imprint with limited resolution. NIL is mainly limited by practicable reasons in imprint area due to the waviness of the substrates. To implement full wafer NIL, the SCIL adopted the composite mold and the sequential imprinting method. The composite mold is composed of two rubber layers on a thin glass support. The in-plane stiffness of the stamp avoids pattern deformation over large areas, while out-of-plane flexibility allows conformal contact to underlying surface features. SCIL is based on a sequential imprinting principle, whereby the soft mold is placed gradually on the substrate and is then removed (see Figure 1). The wavelike progression of the contact front minimizes air inclusions even on large areas, and the sequential separation of the mold and substrate (peel-off demolding) allows for a clean and reliable disconnection that does not damage the patterned structures. Currently, the SCIL has achieved sub-10-nm resolution on wafer-scale areas (6 inch wafer). The SCIL technology can well cope with non-flat substrates and implement full wafer nanoimprinting in a single step. For the SCIL process, the
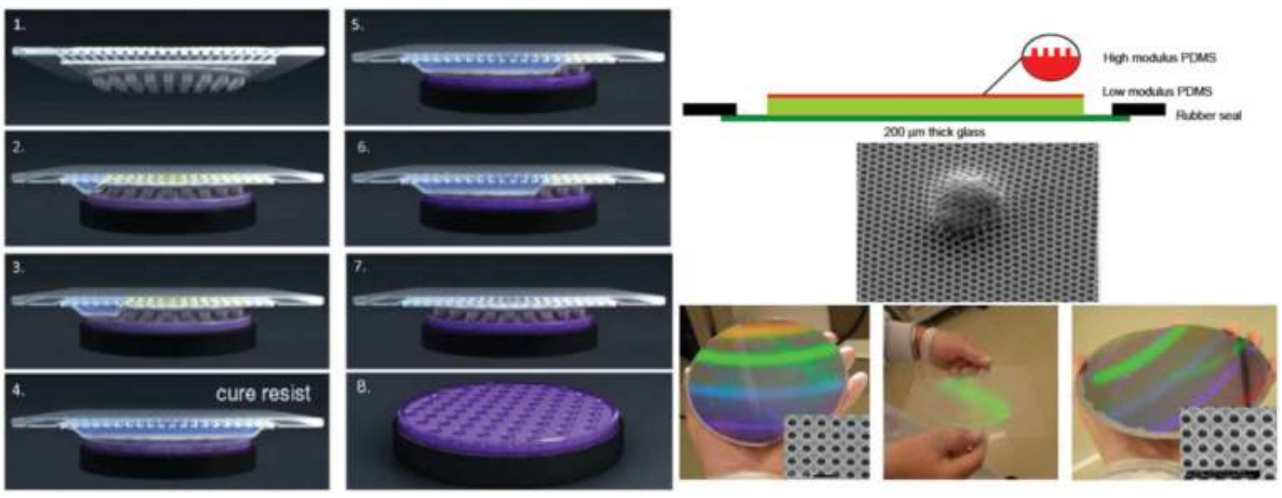

Figure 1. Schematic diagram of substrate conformal imprint lithography (SCIL), composite mold, and photo of imprinted patterns $[1,18]$. 
curing of sol-gel resist relies on the diffusion of solvents into the PDMS stamp. Depending on the operation and preparation conditions, the curing time varies from 5 to $15 \mathrm{~min}$. In order to shorten curing time of the process, a UV-enhanced SCIL process using UV curable material has been developed. Fader et al. introduced UV-SCIL with purely organic UV-curing materials showing curing times of $17 \mathrm{~s}$ [1, 18-19]. The SCIL technology can well cope with non-ideal substrates and implement full wafer imprinting in a single step. The excellent performance of SCIL in respect to substrate conformity and pattern fidelity over large areas enables this nanoimprint technology which is a powerful tool, especially for applications like LED/VCSEL, photonic crystal LEDs, three-dimensional (3D) photonic structures, nano-patterned sapphire substrates, optical elements, or patterned media [1].

Lan et al. reported a full wafer soft UV-NIL using a composite mold with tri-layer structure. The composite mold comprises of a thin layer of fluoropolymer-based material as the patterning layer, a thick layer of s-PMDS as intermediate flexible or cushion layer, and a thin PET (or glass sheet) as the support layer. Figure 2 illustrated the schematic diagram of the proposed full wafer soft UV-NIL process. The imprinting process is performed by a sequential and micro-contacting solution starting from the center to two sides of the mold. The separation process employs a continuous 'peel-off' demolding mode starting from two sides to the center of the mold. Compared to the SCIL, the distinct advantages of the process include: (1) the imprinting and demolding procedure take the mold center as axis of symmetry, are carried out at the same time

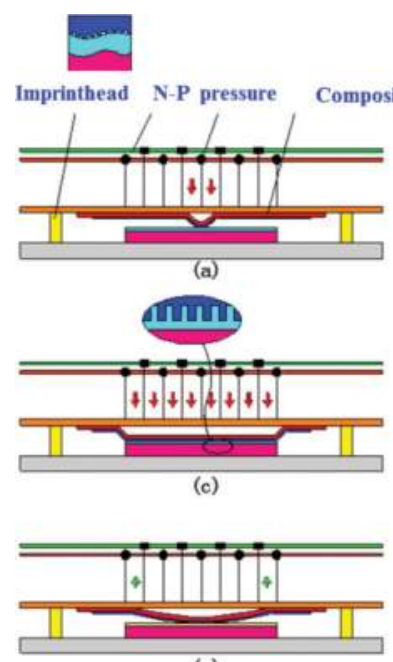

(e)

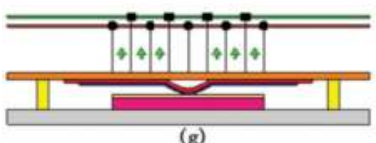

(g)

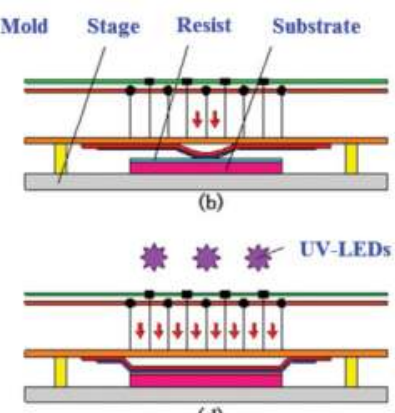

(d)

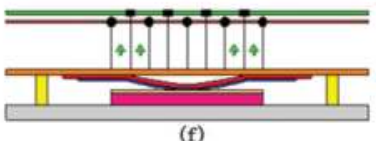

(f)

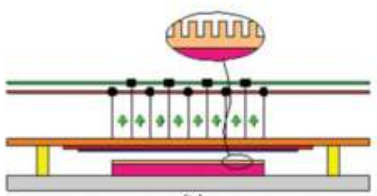

(h)

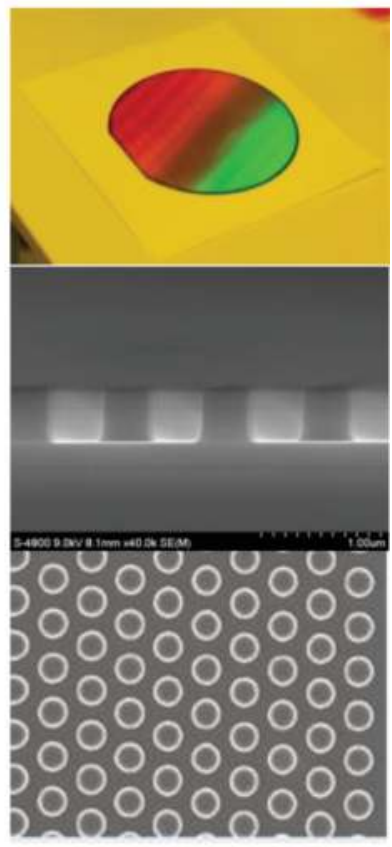

Figure 2. Schematic of full wafer nanoimprint lithography with a tri-layer composite mold and imprinted patterns (a-h). 
on two sides with higher throughput and easier eliminating trapped air bubble; (2) an enhanced demolding approach is adopted; and (3) since the imprinting procedure is performed under a low vacuum pressure environment, it can better remove the trapped air bubbles and provide completely conformal contact [11].

Lan et al. proposed a new full wafer soft UV-NIL based on two air chambers. The imprinting and demolding processes are conducted by the close collaboration between the switching positive pressure and negative pressure in two air chambers and the upward and downward movement of substrate stage. Figure 3 illustrated the basic principle and work flowchart of the proposed full wafer UV-NIL process and imprinted patterns. Compared to other full wafer soft UV-NIL, it has several outstanding advantages such as more compact configuration, easier operation, higher yield rate, easy to control, etc.

By integrating the imprint technology and roll-to-roll technology, which is based on extruder's coater technology, Toshiba Machine developed a nanoimprint equipment ST50S-LED for highbrightness LEDs. Figure 4 shows the schematic diagram of the UV imprinting equipment. This imprinter enables UV imprint on the whole surface of a curved wafer in film mode, and has the features of high-precision control of the press position and press force. To support imprinting on a warped substrate or depositing defect substrate, a flexible resin mold is used in order to enable imprinting with high accuracy of form and less dispersion of residual resin layer. The rolled resin mold made by the roll-to-roll UV imprinting machine (CMT series) is used for automatic successive imprinting. A ST Head is employer to prevent un-uniform contact caused by parallelism difference between the substrate and mold to enable uniform transcription on the surface. This can enable automatic whole-surface UV imprint to form a fine-shape pattern of nanometer-order on a curved LED substrate. The maximum throughput of this equipment is 45 wafers/h [20, 21].

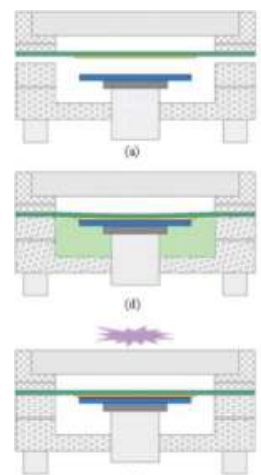

(a)

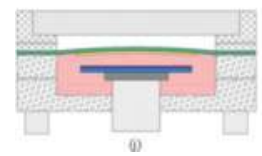

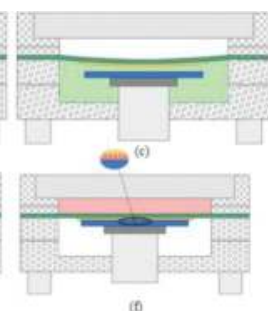

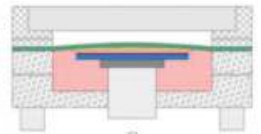

6)
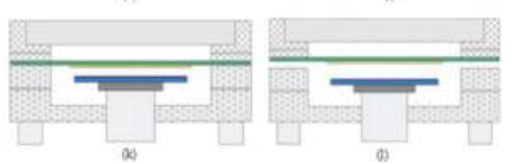
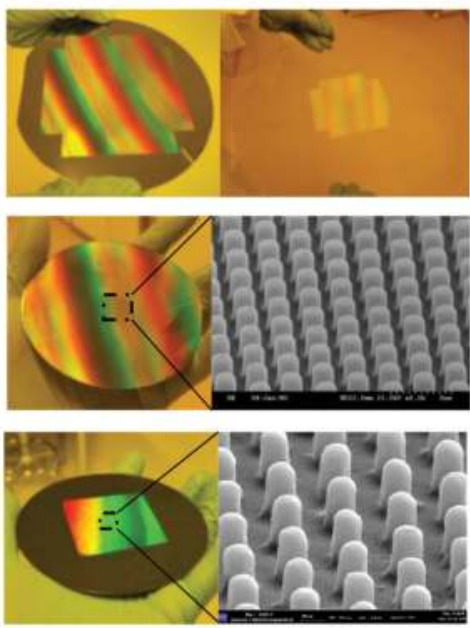

Figure 3. Schematic diagram of the principle of full wafer nanoimprint lithography with dual chambers, and imprinted patterns (a-l). 

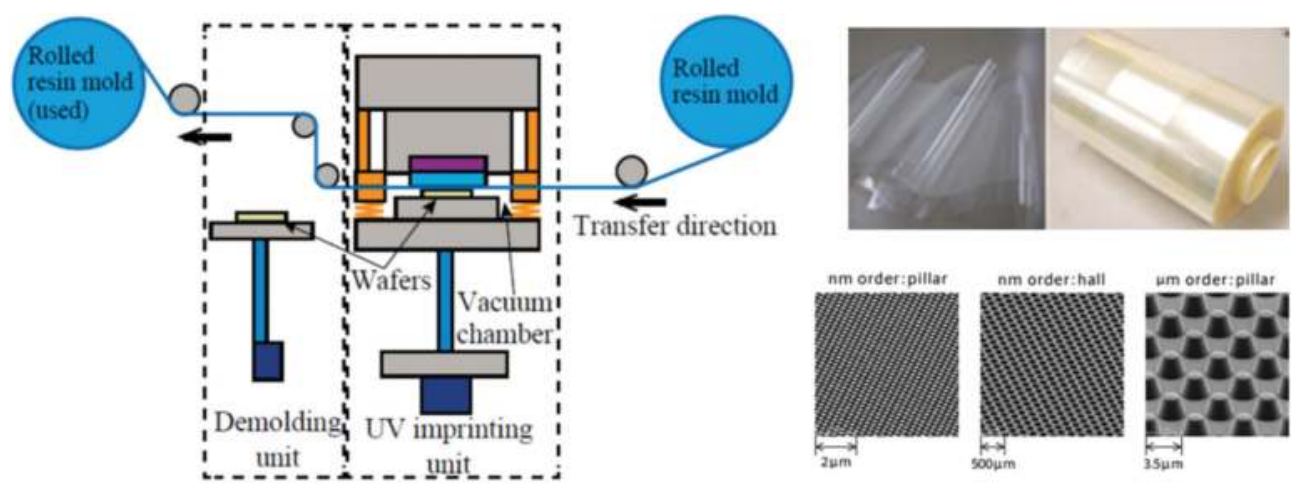

Figure 4. Schematic diagram of the UV imprinting equipment, resin mold (roll type) and imprinted patterns [20, 21].

A roller press mechanism is also used to in full wafer NIL process. Since a roller press mechanism is utilized, the actual contact area during imprinting is only a line along the roller in contact with the substrate rather than the entire mold area. Furthermore, the roller-based NIL process has the advantage of reduced issues regarding trapped air bubbles, thickness variation, and dust pollutants, which also greatly improve its replication uniformity. Youn et al. reported the prototype development of a roller-based imprint system and its application to large-area polymer replication for a microstructured optical device. Figure 5 demonstrated its basic principle and process layout. A roller is utilized to press a flat flexible mold supported by several coil springs onto the polymer substrate. As the roller imprints onto the substrate via platform movement, pullers will be automatically elevated to lift and separate the flexible mold from the substrate. The system has the capacity to replicate ultra-precision structures on an area of $100 \mathrm{~mm} \times 100 \mathrm{~mm}$ at the scanning speed range of $0.1-10 \mathrm{~mm} / \mathrm{s}$. Feature sizes down to $0.8-5 \mu \mathrm{m}$ have been reported to be successfully imprinted [15, 22].

ESCO in Japan developed a Roller Press Scan ${ }^{\circledR}$ method (pressing sequentially using a roller), as shown in Figure 6. A roll is utilized to directly press the substrate that is placed onto resincoated mold rather than the flexible mold. The maximum imprint area is $450 \mathrm{~mm} \times 500 \mathrm{~mm}$. The process has been applied to many fields such as moth-eye sheet, photonic crystal, optical waveguide (several tens of micrometers), micro-lens array (few millimeters), etc. [23].
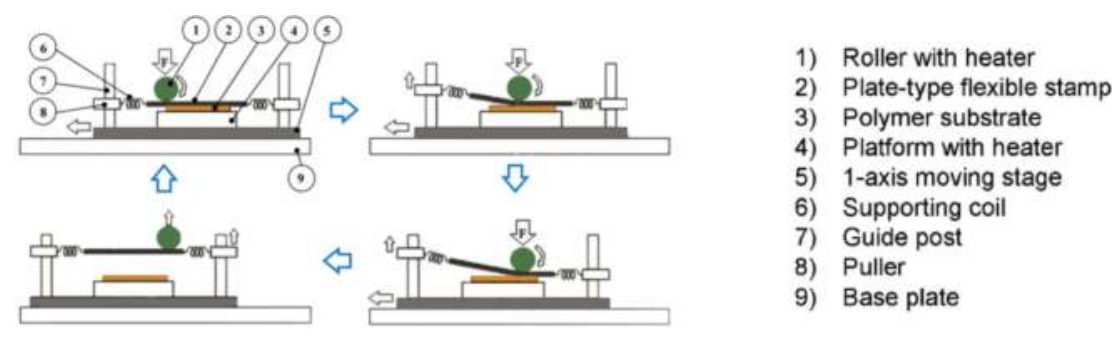

Figure 5. Process flow chart of the roller-based NIL process [22]. 

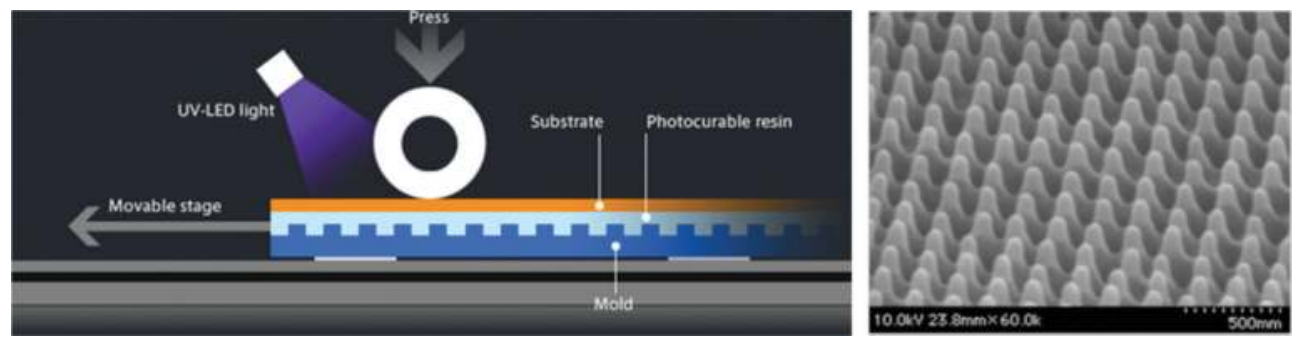

Figure 6. Schematic of the Roller Press Scan ${ }^{\circledR}$ method and imprinted patterns [23].

Soft UV-NIL has been considered as one of the most promising methods for mass producing micro- and nanoscale structures on the entire wafer at low cost for the applications in compound semiconductor optoelectronics and nanophotonic devices, especially for LED patterning.

Traditional plate-to-plate type NIL using a large mold is the simplest way for producing largearea nanostructures, but commonly requires high pressure that increases the possibility of mold deformation even the damage of the mold and the substrate. Further, the thickness variation on a mold or substrate can be as large as a micrometer on a large wafer area, and it is hard to be compensated. During imprinting, non-conformal contact occurred by the local flatness distortion in the mold causes the reduction of replication uniformity and leads to a huge stress concentration, resulting in the warping or distortion of the stamp. Therefore, soft UV-NIL by using a flexible mold has been proven to be a cost-effective high volume nanopatterning method for large-area structure replication up to wafer-level (up to $300 \mathrm{~mm}$ ) in the micrometer and nanometer scale, fabricating complex 3D micro/nanostructures, especially making large-area patterns on the non-planar surfaces even curved substrates at low cost and with high throughput.

As a result, in order to better implement the full wafer NIL, some critical issues and promising solutions are described as follows. For the imprinting process, four important issues must be considered: (1) achieving uniform pressure distribution across the full wafer; (2) ensuring entirely conformal contact on the imprinting full field; (3) avoiding trapped air bubble defects; and (4) reducing imprinting forces. Applying gas-assisted press, sequential contact, vacuum imprint environment, and composite flexible mold may be effective solution. For the demolding process, peel-off demolding, fluoropolymer-based mold, and PVA-based thin-film mold can be used to avoid demolding defects and mold damage. For full wafer NIL, how to fabricate a large size wafer-level master has been became as the most challenging issue.

\subsection{Roller-type NIL}

In full wafer NIL (P2P), the entire imprint area is imprinted in a single imprinting cycle regardless of its size. However, this method is unsuitable for very large imprinting areas as it would require especially large imprint force, which may reach $20 \mathrm{kN}$ of force for an 8 -in. wafer [15]. The roller-type NIL (RIL) provides a unique solution to these challenging issues encountered in the conventional wafer-scale NIL process, because only a line area is in contact during imprinting, thus requiring a much smaller force to replicate the patterns. Moreover, because 
the stamp used in R2R NIL is in the form of a roller shape, the stamp-substrate separation proceeds in a "peeling-off" mode, which requires very small demolding force.

The roller-type NIL (RIL), first proposed by the Chou group [24], has now become one of the most promising candidates for rapid patterning on a very large-area substrate. Typical RNIL has a roller-shaped mold which contacts to a counter roller. A substrate goes through it and micro/nanostructures are transferred from the roller-shaped mold to the substrate. This technique can realize continuous imprinting, and can transfer patterns to the rigid or flexible substrates. Compared to P2P NIL, RNIL has the advantages of better uniformity, a lower imprint force, and the ability to repeat the patterning process continuously on a large substrate. Additionally, due to the line contact, the roller-based NIL process has the advantage of reduced issues regarding trapped air bubbles, thickness variation, and dust pollutants, which also greatly improve its replication uniformity.

\subsubsection{Roll-to-plate type NIL}

The roll-to-plate (R2P) NIL process uses a roller mold to pattern large-area rigid substrate. A roller mold is employed to imprint various rigid substrates such as glass substrates. The typical applications include flat panel displays, solar cells, anti-reflective glasses, etc. In 1998, Steven Chou's group firstly demonstrated a R2P thermal NIL process. A roller mold, which was made by wrapping a $100 \mu \mathrm{m}$ thick nickel compact disk master mold with sub-micron features around the smooth roller, is imprinted and rolled on the substrate. Sub-100 nm resolution pattern transfer has been achieved using this method [24].

Ahn et al. described a R2P UV-NIL process for fabricating large-scale nano- and micropatterns on rigid substrates, as shown in Figure 7. The UV roll nanoimprinting system has the following components: a dispensing unit, a pair of flattening rollers, a UV light illumination unit, gap

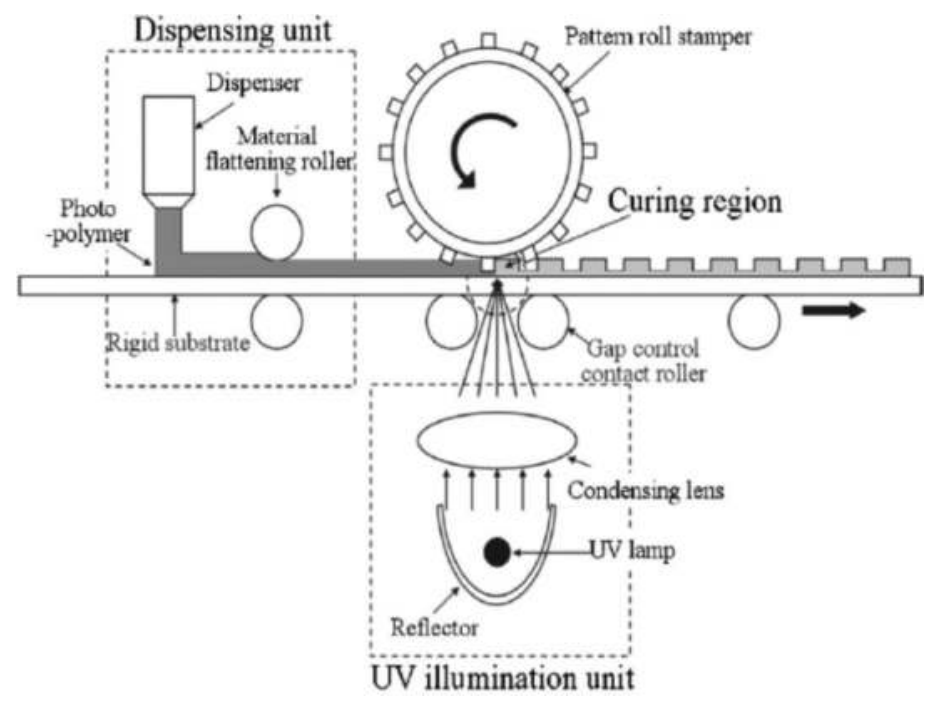

Figure 7. Schematic of R2P UV-NIL process for a rigid and transparent substrate developed by Ahn et al. [25]. 
control contact rollers, and a roller mold. The pair of flattening rollers enables the uniform coating of the photopolymer onto the substrate, which is achieved by controlling the size of the gap between the rollers. To obtain thickness uniformity, passive gap control by pressing the contact roller against the roll stamper was used. However, there is a limitation of the process since the substrates used should be transparent [25].

Park reported a R2P UV-NIL tool and process using double-layered soft cylindrical stamp to replicate nanoscale patterns on a Si substrate, as shown in Figure $8.30 \mathrm{~nm}$ dot array patterns have been obtained. The R2P UV-NIL tool consists of a transparent quartz cylinder controlled by two synchronized motors, a passive compliant stage, a pressing unit in the $\mathrm{Z}$ direction, a UV system with a wavelength of $365 \mathrm{~nm}$, and a fine stage with $3 \mathrm{~nm}$ resolution. Because of the synchronized motors used, there is no slip or misalignment between the replica and the quartz cylinder due to the tight fitting and compliant stage of the imprinting process. The trapped air problem is continually eliminated by means of the line of contact between the cylinder and the flat substrates from the first contact position to the last contact position. Ahn and Guo demonstrated large-area (4 in. wide) continuous imprinting of nanoscale structures by using a newly developed 6 in.-capable roll-to-plate (R2PNIL) apparatus. The grating patterns with $300 \mathrm{~nm}$ line width have been continuously transferred on glass substrates with greatly enhanced throughput [26].

Hitachi, Ltd. developed a sheet nanoimprint technology which enable 100 times higher productivity of nanoscale patterns, as shown in Figure 9. The enormous productivity is enabled by continuous processing of heating, pressing, cooling and separation using belt-shaped nanomold. A $200 \mathrm{~nm}$ in diameter and $240 \mathrm{~nm}$ tall dots (aspect ratio 1.4) were formed directly onto a $15 \mathrm{~m}$-long polystyrene film. The process has some outstanding advantages such as extending cooling time, without size limitation of the mold [27].

In order to achieve transferred micropatterns with a precise profile yet no residual layer on the substrate, a roller-reversal imprint (RRI) process was proposed as shown in Figure 10. The resist is dispensed onto the roller mold using slot die instead of being coated onto the substrate, allowing it to fill in the mold cavities. A doctor blade is employed to remove excessive resist from the roller mold as it rotates. Upon contact with the substrate, the resist is transferred onto the substrate in a similar manner to a gravure printing. The transferred resist will then be
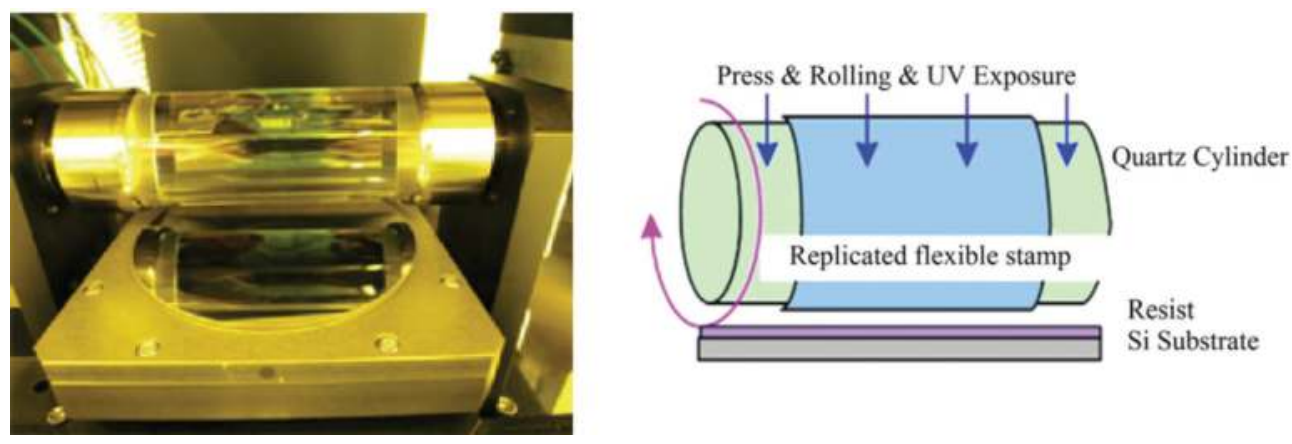

Figure 8. R2P UV-NIL using double-layered soft cylindrical stamp [26]. 

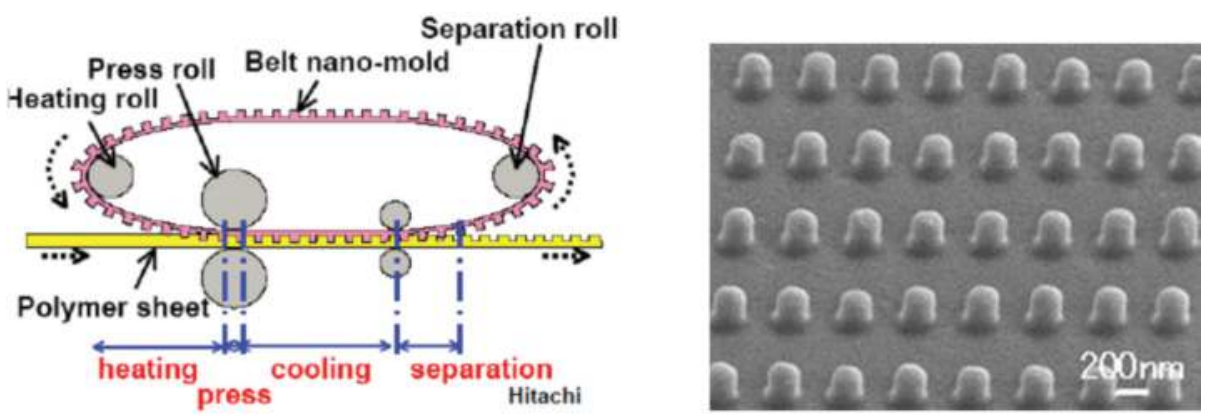

Figure 9. Schematic of sheet NIL developed by Hitachi and imprinted high aspect ratio pattern [27].

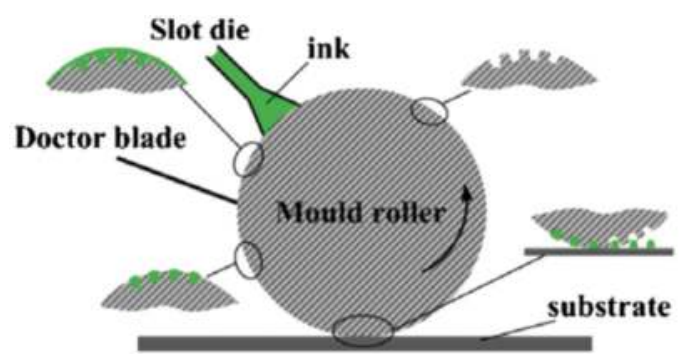

Figure 10. Schematic diagram of roller-reversal imprint process [28].

solidified by either UV or thermal curing. Feature sizes ranging from 20 to $130 \mu \mathrm{m}$ in line width and $10-100 \mu \mathrm{m}$ in depth have been successfully patterned using the roller-reversal imprint method [28].

While R2P NIL methods have great advantages over conventional P2P NIL in terms of imprint force, throughput, and size of equipment, it still has several limitations in realizing a continuous imprinting process.

\subsubsection{Roll-to-roll type NIL}

For the R2R NIL process, a roller mold is utilized to imprint onto a flexible substrate (or resistcoated flexible substrate) on a supporting roller instead of a flat plate in R2P NIL processes. The entire process is based on the roll-to-roll manufacturing concept, which has the advantages of continuous process, large-area patterning, and high throughput. It provides a highly promising solution for industrial scale applications. Therefore, the continuous R2R NIL technique offers a promising solution for high-speed large-area nanoscale patterning with greatly improved throughput. Moreover, it can overcome the challenges faced by conventional NIL in maintaining pressure uniformity and successful demolding in large-area imprinting [5, 15]. R2R NIL processes can be generally separated into two groups: thermoplastic roller imprinting and UV-curing roller imprinting. 


\subsubsection{R2R thermal NIL}

Continuous imprinting of thermoplastics with a roller mold is a promising technique because of its simplicity in terms of tool setup and materials. All that is required for patterning is adequate heat and mechanical pressure, a suitable roller mold and a thermoplastic web that can be patterned directly; thus avoiding the need to deposit a patterning medium (resist) onto a substrate and all the issues arising from the additional processing steps. In addition, the technique is flexible in terms of materials and substrate selection. Various materials for the roller mold and a wide variety of thermoplastic materials can be used as either cast films on a substrate or as a stand-alone web [5]. In addition, functional materials such as semiconducting or light-emitting polymers can be directly patterned with this technique. In particular, with a roller mold seamless patterning is achievable and this is very useful for large-area low cost manufacturing applications such as anti-reflection coatings, micro-lens arrays and wire grid polarizers (WGPs).

Existing investigations on thermal roller imprinting revealed poor pattern transfer fidelity, especially for high aspect ratio features. The traditional $R 2 R$ imprinting process suffers from the lack of an effective holding and cooling stage so that the adverse effects from the viscoelastic nature of polymers are not managed. To solve this problem and further improve the production rate, a new extrusion roller imprinting process with a variotherm belt mold was proposed, as shown in Figure 11, and its prototype was also developed at a laboratory scale. The major components of the R2R tool include an extruder, a belt mold, a roll-to-roll setup, and an induction heating unit. The extruded polymer film is imprinted between the belt mold and the pressure roller. Due to the variotherm capability, the imprinted film is effectively cooled before it is released from the belt mold. The experimental results demonstrated that a $30 \mu \mathrm{m}$ sawtooth pattern can be faithfully transferred to extruded polyethylene film at take-up speeds higher than $10 \mathrm{~m} / \mathrm{min}[29]$.

3D and multilayered nanostructures are becoming important with the technological advances in nanodevices such as nanoelectromechanical systems, nanofluidic devices, and nanophotonics. Nagato et al. from University of Tokyo proposed an iterative roller imprint method of multilayered nanostructures by combination of imprinting and bonding process. Thermoplastic polymer film is imprinted using a nanostructured mold and heated rollers (first layer). The next imprinted thin film is thermally bonded on the backside of the first layer using other rollers. By repeating these processes, a multilayered nanostructure was fabricated. By this method, multilayered
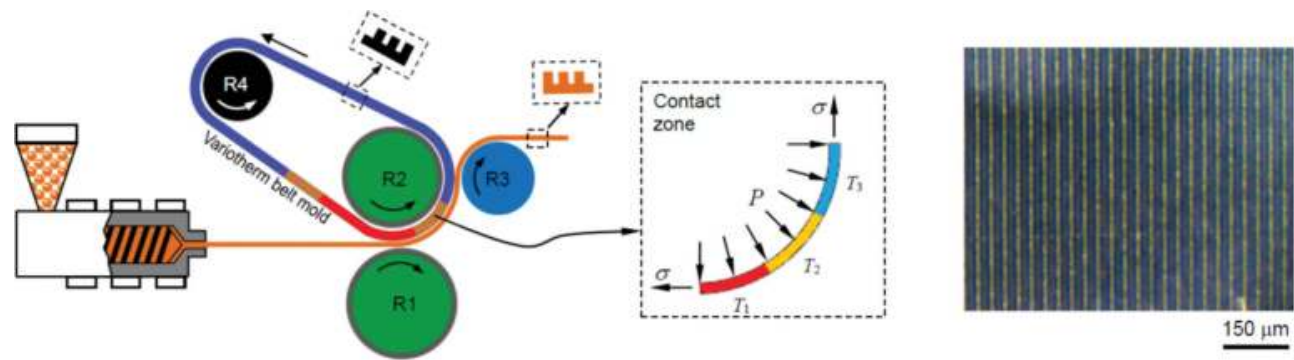

Figure 11. Schematic of extrusion roller imprinting process with a variotherm belt mold, imprinted structures [29]. 
nanostructures with a pitch of $800 \mathrm{~nm}$ and a depth of $300 \mathrm{~nm}$ were fabricated using PMMA. Figure 12 illustrated the iterative roller imprint method of multilayered nanostructures and imprinted multilayered nanogaps with five-layered nanostructures [30].

In 2013, EVG releases the industry's first roll-to-roll thermal NIL tool (EVG $\left.{ }^{\circledR} 570 R 2 R\right)$ which can mass produce films and surfaces with micro- and nanometer-scale structures for a variety of medical, consumer, and industrial applications, including microfluidics, plastic electronics, and photovoltaics. Recently, an EVG750 ${ }^{\circledR}$ R2R hot embossing system was released, as shown in Figure 13. The EVG750R2R's innovative design provides excellent temperature and pressure uniformity for micro- and nanoscale patterning on a broad range of materials. It is designed to deliver the highest flexibility for R\&D applications with a clear vision for automated mass manufacturing of flexible devices [31].

There are two challenging issues for R2R thermal NIL processes. One issue related to the imprinting of thermoplastic materials generally is the flow behavior in the vicinity of large or strongly nonuniform mold features. Another issue that must be solved is an effective cooling solution prior to demolding.

\subsubsection{R2R UV-NIL}

Although R2R thermal NIL has the advantage of simplicity and flexibility in terms of tool setup and materials, the process involves relatively high pressure (typically at least $5 \mathrm{MPa}$ ) and high temperature (typically about $100-300^{\circ} \mathrm{C}$ ) due to the high viscosities of thermoplastic materials imprinted. Unlike with the imprinting of thermoplastic materials with R2R thermal NIL, R2R UV-NIL does not require elevated temperature or large applied pressures to create patterns which has shown higher throughput and lower product cost, because low pressures are used in UV roller imprinting, it is easier to protect the roller mold from accumulating defects due to particles and residues; thus improving mold lifetime and the overall quality of
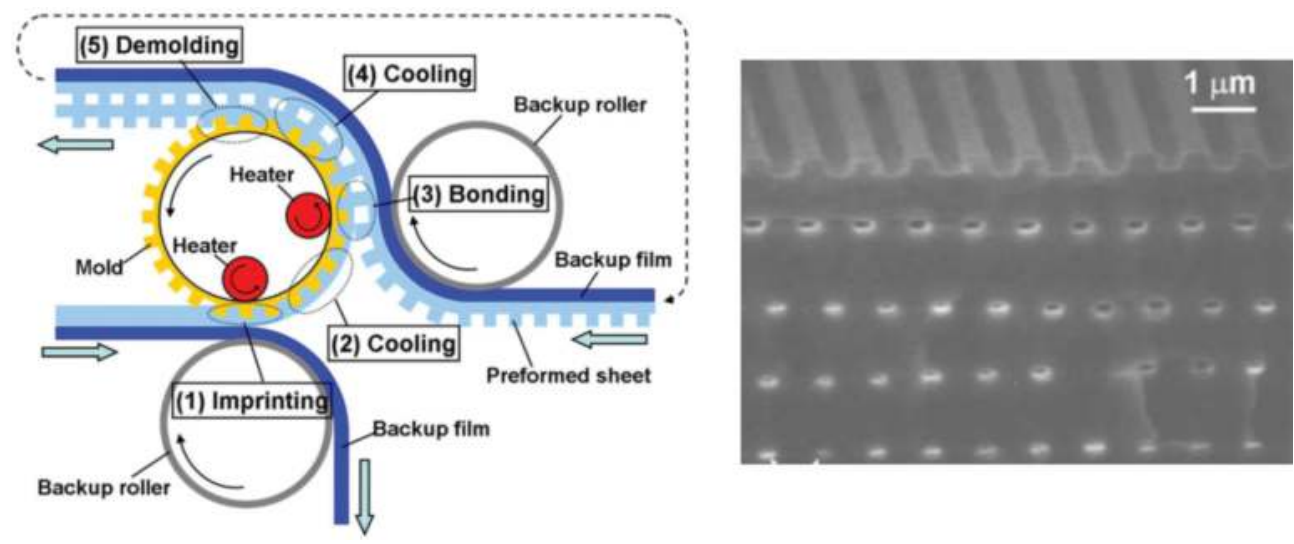

Figure 12. Schematic of roller imprint system for multilayered nanostructures, imprinted multilayered nanogaps with five-layered nanostructures [30]. 

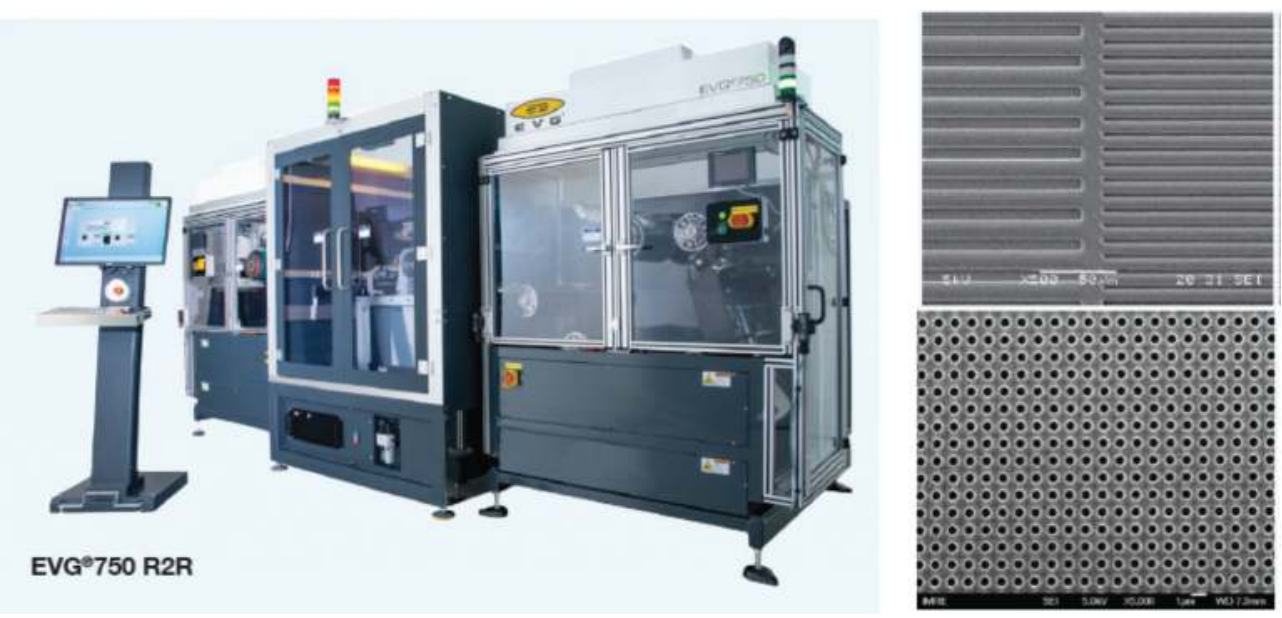

Figure 13. Photo of the EVG750 ${ }^{\circledR}$ R2R hot embossing system, imprinted, SEM image of $10 \mu \mathrm{m}$ and $30 \mu \mathrm{m}$ microfluidic structures replicated into PMMA, $500 \mathrm{~nm}$ holes replicated into PMMA [31].

replication [5]. In addition, low pressure requirements enable the use of soft mold materials such as ETFE, PDMS without risk of feature distortion.

Figure 14 illustrated a typical R2R UV-NIL system. It mainly consisted of the following functional components such as a dispensing unit, a doctor blade (or a pair of flattening rollers), a UV source unit, a roller mold, pressure rollers, a demolding roller, etc. [5]. A dispensing system is utilized to deposit a UV curable liquid resin either as a pattern of drops or as a continuous film. Following deposition of the resin, a variety of thickness control measures can be employed, such as a doctor blade. Multiple pressure rollers are often used to ensure

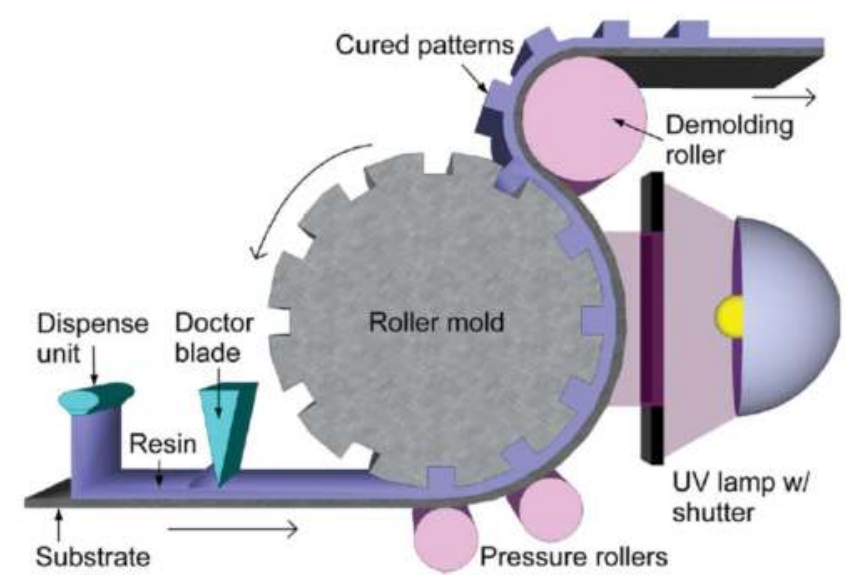

Figure 14. Schematic of a typical R2R UV-NIL system [5]. 
uniform spreading of the resin and filling of the roller mold cavities prior to UV exposure. A demolding roller is used to peel the cured patterns off the roller mold. How to extend roller mold lifetime is a challenging issue for the real commercialization or industrial applications of roller-type NIL. The roller mold should have good wear resistance properties. The mold material used should have low surface energy to ensure that the resist does not adhere to the mold surface during the demolding process, and to reduce friction during imprinting. A release coating on the roller mold surface can effectively prolong roller mold lifetime by preventing the adhesion of the resists. However, this release coating gradually deteriorates as the number of repetitions of NIL transfer increases. It is therefore important to maintain the lifetime of the release agent. A roller mold generally need be coated with a fluorinated silane anti-sticking layer to prevent sticking problems. In addition, mixing of various types of release agent is also an effective way in increasing the lifetime of the roller mold [15].

Guo et al. presented a R2R UV-NIL process for the fabrication of metal wire grid polarizers with nanoscale structures (down to $70 \mathrm{~nm}$ line width and $100 \mathrm{~nm}$ pitch) on a flexible substrate. Figure 15 demonstrated the schematic of the R2R UV-NIL process for fabricating metal wire grid polarizer and imprinted patters. The system is composed of three functional modules: coating module, imprinting module, and metal deposition module. An ETFE flexible fluoropolymer stamp wrapped around a stainless steel roller is adopt to be the roller mold for the UV R2R NIL. The benefit of using ETFE is that it has good anti-stick properties (critical surface tension of $15.6 \mathrm{mN} \mathrm{m}^{-1}$, cf. PDMS $19.6 \mathrm{mN} \mathrm{m}^{-1}$ ) that do not deteriorate over many imprint cycles or from exposure to UV light, unlike fluorinated SAM anti-stick coatings [32].

A self-aligned imprint lithography (SAIL) was proposed by HP for solving alignment problem in R2R imprinting process, as shown in Figure 16. The SAIL has been used to fabricate precision electronic devices that require multiple alignment steps on a large dimensionally unstable substrate by incorporating a single imprinting step. The technique solves the problem of precision interlayer registry on a moving web by encoding all the geometry information required for the entire patterning steps into a monolithic three-dimensional imprint with
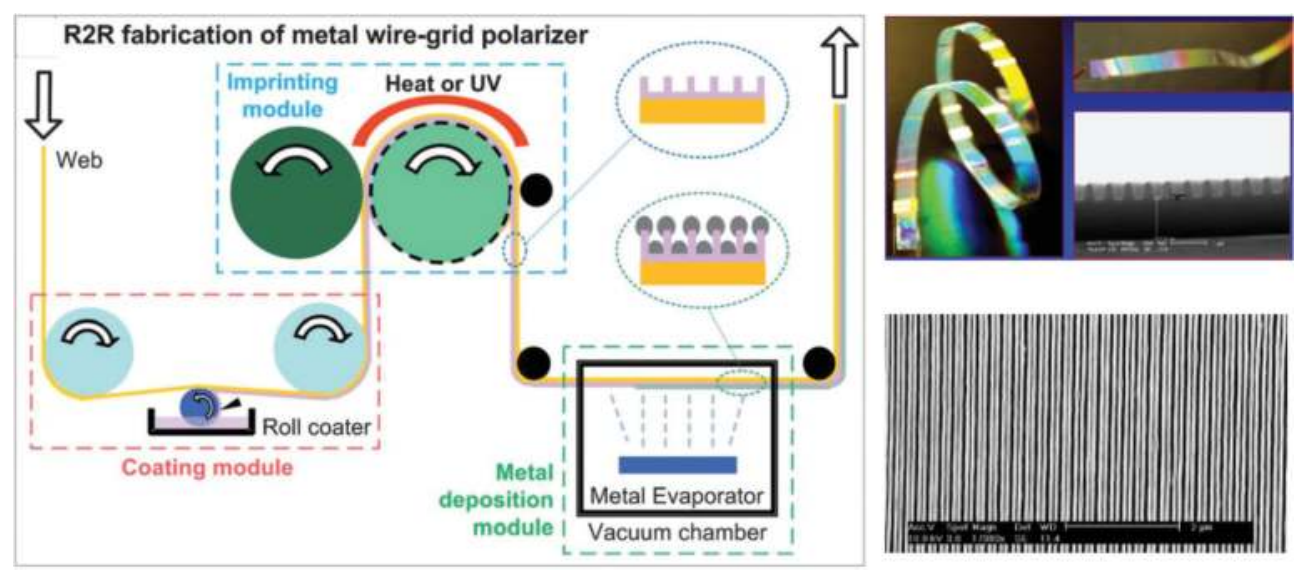

Figure 15. Schematic of R2R UV-NIL process for fabricating metal wire grid polarizer and imprinted patters [32]. 

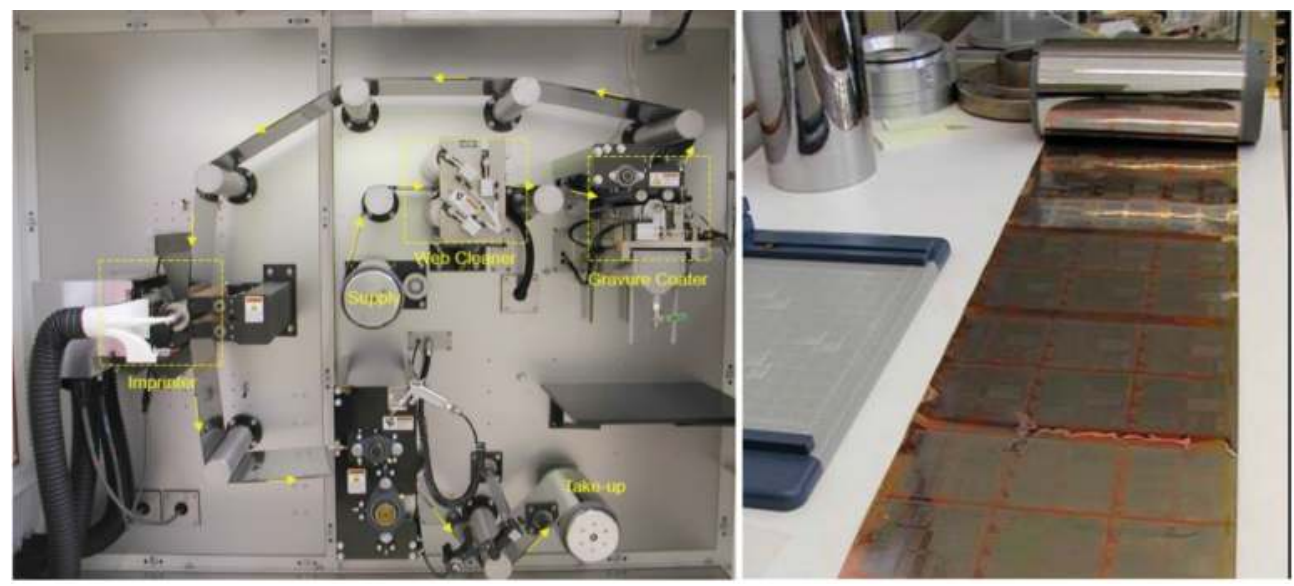

Figure 16. Photo of roll-to-roll coating and imprinting apparatus, imprinted active-matrix backplanes [33].

discrete thickness modulation. It can enable the patterning and alignment of submicron-sized features on meter-scaled flexible substrates in the R2R environment. The SAIL provides an effective way for mass producing flexible electronics and flexible display backplanes in particular for commercial production [33, 34].

Due to the limitations of current roller mold materials used for R2R NIL, John et al. described a large-area, continuous R2R nanoimprinting with PFPE composite molds. A R2R Nano Emboss 100 was also developed using the PFPE composite molds to address the challenge of fabricating nanostructured thin films on a high-speed, high-reliability platform. The tool consists of five sections, namely unwind, coating, imprinting, metrology/coating, and rewind, as shown in Figure 17. John et al. showed the efficiency, reliability, and durability of the PFPE-based
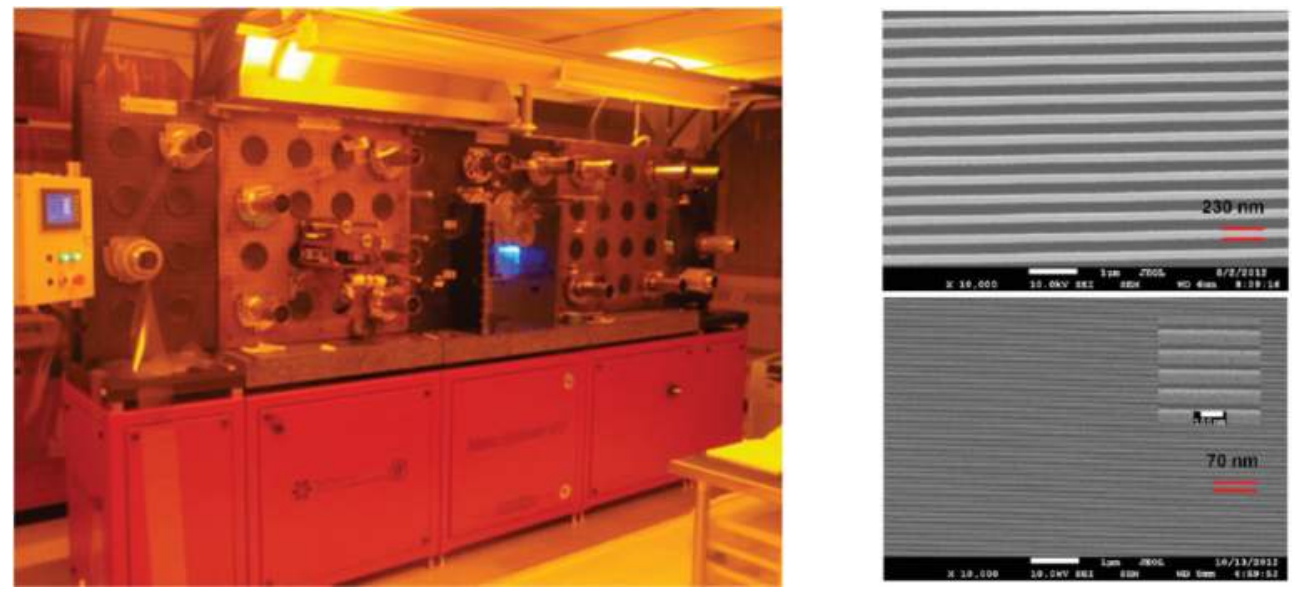

Figure 17. Photo of the custom designed R2R nanoimprinter; SEM images of 230 and $70 \mathrm{~nm}$ imprinted gratings on PET [35]. 
composite molds in replicating 1D and 2D micron to sub-100 nm features with high fidelity over successive imprinting cycles in a dynamic large-area high-speed roll-to-roll nanoimprinting process [35]. The experimental results have exhibited that the PFPE composite mold replicated nanofeatures with high fidelity and maintained superb mold performance in terms of dimensional integrity of the nanofeatures, nearly defect-free pattern transfer and exceptional mold recovering capability throughout hundreds of imprinting cycles [35].

A roll-to-roll type UV imprinting machine has been developed for mass producing resin molds, wire grid polarizers, flexible solar cells, etc. Figure 18 illustrated the schematic of the R2R UVNIL and imprinted products. An "intermittent coating" technology employing a gravure roll to mold UV curable resin coated on film is introduced. Sub-100 nm patterns, especially sub-30 nm patterns, onto ultraviolet (UV) curable resin on film substrate have been obtained. They also have investigated the potential of R2R UV-NIL for future wearable devices that would require large-area nanopatterning. This result indicates that the process has the potential to make sub$30 \mathrm{~nm}$ patterns on film [36].

Ahn and Guo illustrated large-area (4 in. wide) continuous imprinting of nanogratings by using a developed apparatus capable of roll-to-roll imprinting (R2R NIL) on flexible web and roll-toplate imprinting (R2PNIL) on rigid substrate. For the proposed process, a belt-type mold is wrapped around two rolls. Either a flexible or rigid substrate coated with a resist mounted on another roll or a flat conveyer is then fed into the contact zone where a mold continuously imprints the replica pattern onto the substrate under conformal pressure [37]. The instant UV curing is followed at the outlet of the mold-substrate contact zone to finish the patterning. The $300 \mathrm{~nm}$ line width grating patterns can be continuously transferred on either glass substrate (rollto-plate mode) or flexible plastic substrate (roll-to-roll mode) with greatly enhanced throughput. Figure 19 showed the R2R NIL process and fabricated products [16, 35].

One significant advantage of the R2R NIL technique is that it inherited the high resolution pattern fidelity benefit from the traditional NIL technique with a drastically increased throughput. The possibility of defect generation during the mold-substrate separation process is considerably lower in the R2R NIL technique due to the peeling mode in which the substrate
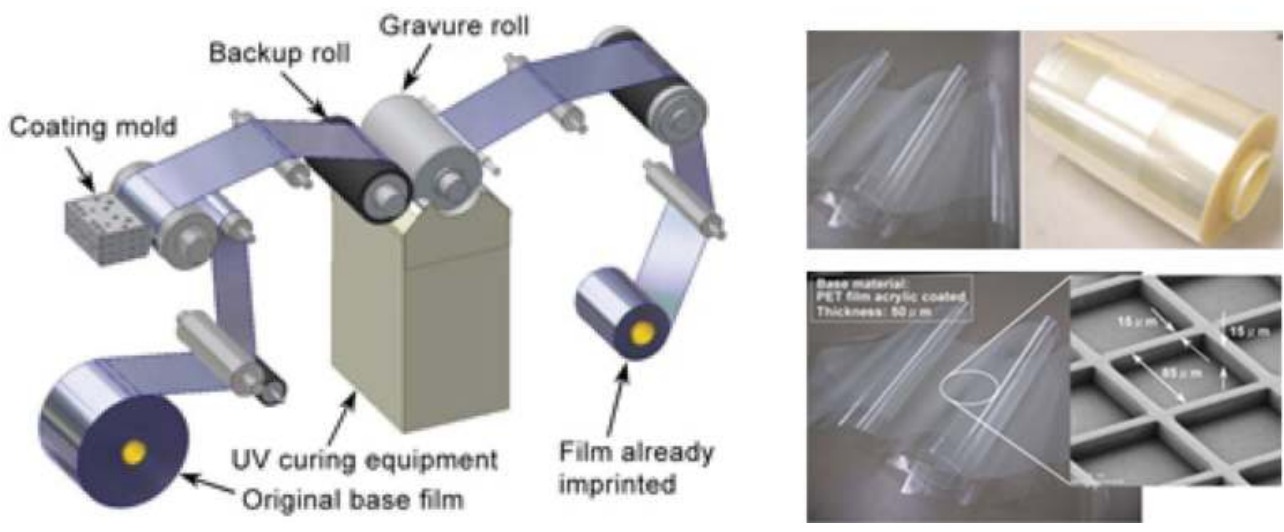

Figure 18. Schematic of R2R UV-NIL NIL developed by Toshiba, imprinted products (resin mold) [36]. 

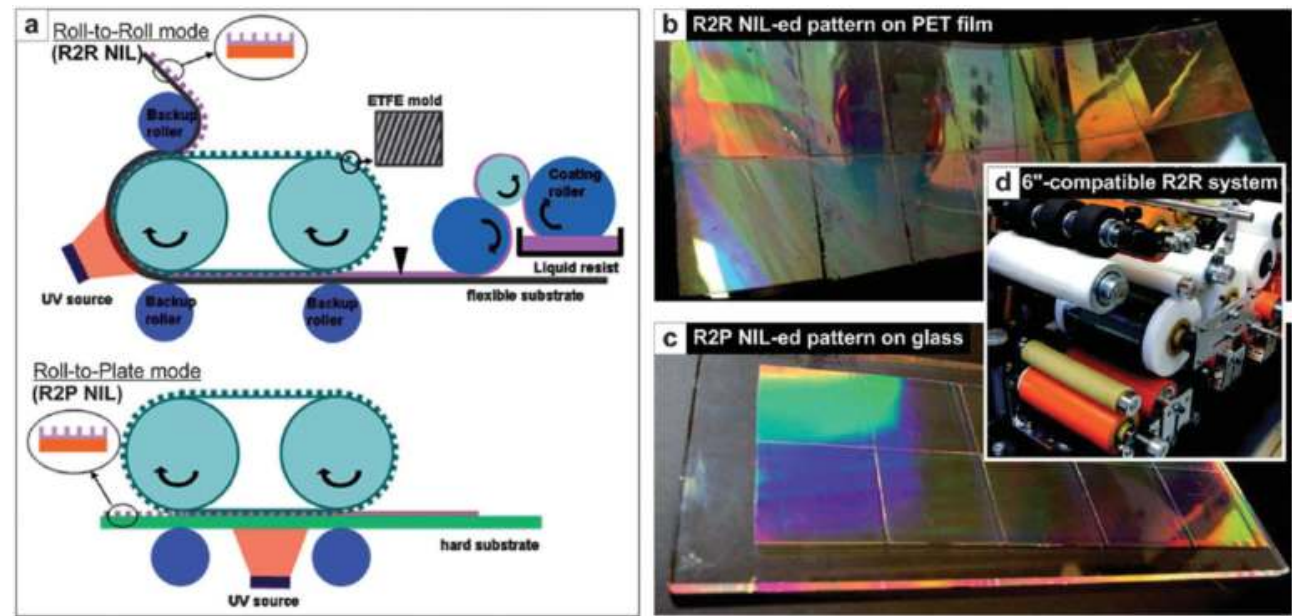

Figure 19. (a) Schematic descriptions of R2R NIL process (top) for flexible substrates and R2P NIL mode (bottom) for rigid substrates; (b) a PET film; and (c) a glass plate [35].

separates from the roller mold assembly as compared to the conventional NIL technique. In particular, R2R NIL has the unique capability of continuous patterning.

\section{Applications of large-area NIL}

Nanostructing has been one of the most promising and enabling technologies to enhance the performance of various products and devices ranging from high-brightness LEDs, high effective solar cells, high density storage to high clearness (HD) display. NIL has proven to be the most cost-efficient way to enable fabrication of nanopatterns on large areas as it is not limited by optical systems and can provide the best pattern fidelity for the smallest structures. Various types of large-area nanostructures and patterns including one-, two- and three-dimensional structures fabricated by NIL have been applied to diverse devices and products to enhance their performances. Large-area NIL technology paves the way for real-world nanotechnology products and innovative applications. Large-area NIL has now been utilized to fabricate various micro/nanostructures and devices for nanoelectronics, optoelectronics, nanophotonics, optical components, nanostructured glass, biological applications, etc. Figure 20 demonstrated representative industrial applications and products with large-area NIL. Moreover, it has become a perfect match for some emerging application fields that are in great need of largearea patterning of submicro and nanoscale features at a low cost, such as patterned magnetic media, high-brightness light-emitting diodes (HB-LEDs), anti-reflective coatings or films, flexible electronics, printed electronics, OLED, wire grid polarizer, flat panel display, microfluidic devices, etc. In particular, this technique has demonstrated great commercial prospects in several market segments, HB-LEDs, anti-reflective coatings or films with moth-eye structures, flexible electronics, solar cells, architectural glass, WGP, optical elements, patterned media, micro-lens arrays, and functional polymer devices [1-5, 38-41]. 


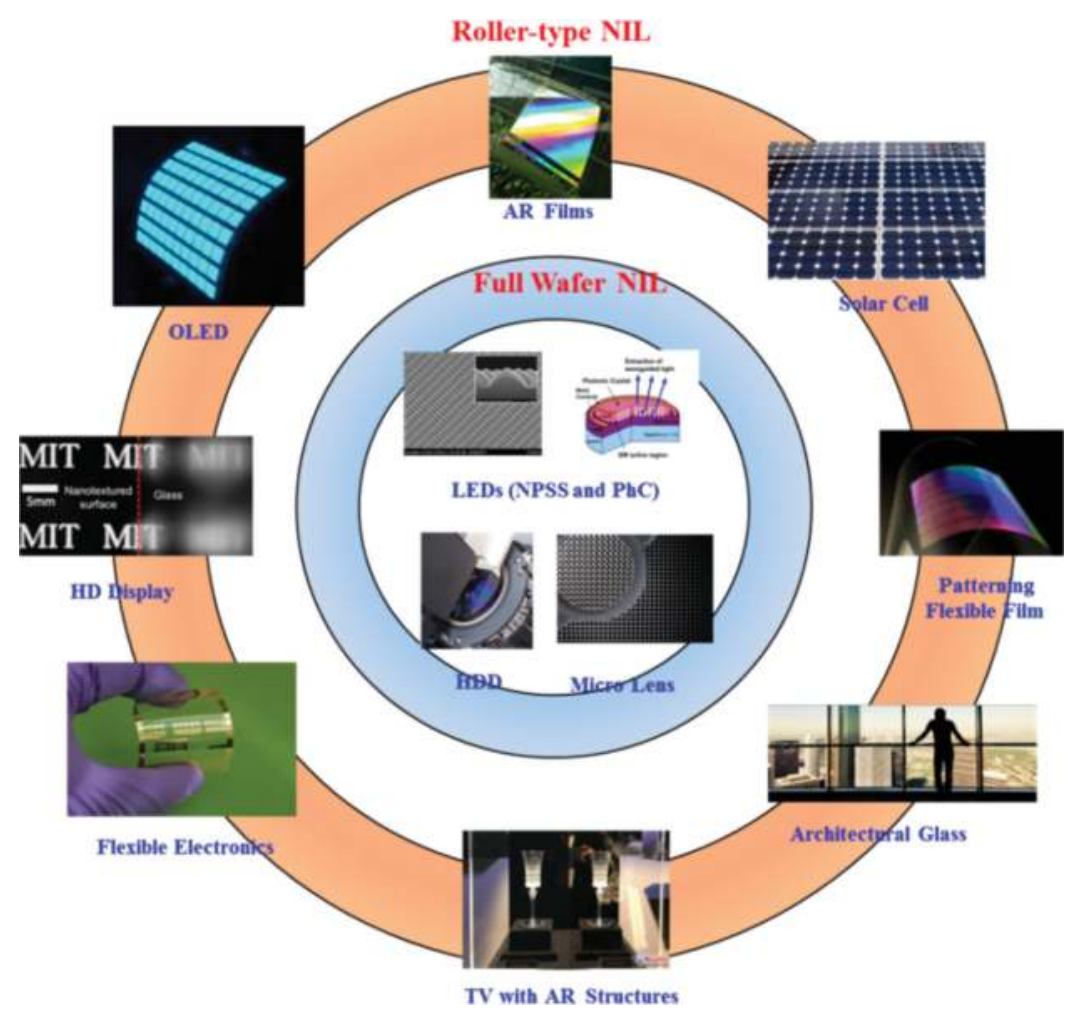

Figure 20. Typical industrial applications and products with large-area NIL.

\subsection{Patterning LEDs}

Light efficiency enhancement and manufacturing cost reduction have always been regarding as the two most crucial issues in LED industry, particularly for the large-scale realization of solid state lighting. Compared with other technologies improving the LED performance, two emerging techniques, photonic crystals and nano-patterned sapphire substrate (NPSS), have shown higher potential in output efficiency enhancement and beam shaping. NPSS and photonic crystal-based LEDs have become the most promising solutions for high-brightness LEDs (HB-LEDs). The typical characteristics of LED epitaxial wafers and sapphire substrates are with large variation in wafer topography (varying TTD), high bow and warp, surface roughness with surface protrusions with micron size and particle contaminations, etc. And these materials tend to be fragile or brittle. Due to the non-planar and rough nature of the LED epitaxial wafer and substrate, existing nanopatterning technologies cannot well meet the requirements of producing these nanostructures in both technology and cost level which mainly originated form the new challenging issues form LED patterning. Due to a very small depth of focus, optical lithography techniques have insufficiently fidelity for LED patterning. As warpage increases with larger wafer sizes, the ability of the photolithography tool to compensate for substrate warpage becomes even more critical. Interference lithography is 
another method of generating periodic patterns over large areas at low cost. Although the patterns made by IL are highly uniform and have superior long-range order, these patterns are usually in very simple geometric forms of grating lines and 2D dots, and their dimensions are difficult to reduce to sub-100 nm due to light diffraction. Furthermore, it is unsuitable for high volume production processes because the optical configuration has to be modified to realize different patterns. In addition, this approach requires a strict control of the environment to maintain stable fringe patterns. Full wafer NIL (soft UV-NIL with flexible mold) has the capability of nanopatterning on non-flat surface over large areas and is less-sensitive to the production atmosphere. Compared to ICs industry, the LED application is much more relaxed than IC's for overlay and defect density. Therefore, full wafer NIL has been considered as one of the most suitable solution for LED patterning. Due to its cost-effectiveness combined with superior processing performance, full wafer NIL will play a crucial role in moving the LED industry into a new realm of nanopattered LEDs with ultra-high efficiency. Figure 21 showed some cases related to LED patterning using full wafer NIL. In addition, some commercial companies such as SUUS, Obducat, EVG, Toshiba, Aurotek, Luminus, etc. have been developing the process and equipment of full wafer NIL for high volume producing PhC LEDs and NPSS [17-20].

\subsection{Photonics (anti-reflective coatings or films, wire grid polarizer)}

Recent developments in many applications, such as photonics, HD displays, and flexible electronics, etc., have observed a rapid increase in demand for a lower cost, higher throughput and higher resolution micro/nanofabrication techniques. The continuous R2R NIL technique offers a promising solution for high-speed large-area nanoscale patterning with greatly improved throughput. In addition, the ability of micro- to nanometer-scale patterning on flexible substrates can enable many new applications in the area of photonics and organic electronics. Some typical applications include anti-reflective coatings and films, wire grid polarizers (WGPs), flexible electronics and flexible display backplanes, nanogratings, light enhancement coatings and films for displays, etc. Perhaps the application which is closest to mass production are anti-reflective coatings and films which are unique in that they are fabricated from homogenous sub-micron moth-eye structures such as cones or pillars. Because they are sub-micron structures and ideally composed of a single material, traditional roll-toroll methods such as flexography or gravure printing cannot be employed. Continuous R2R UV-NIL is ideally suited, especially if the refractive index of the substrate web and the UV curable resin can be closely matched over the visible light spectrum. One of the most
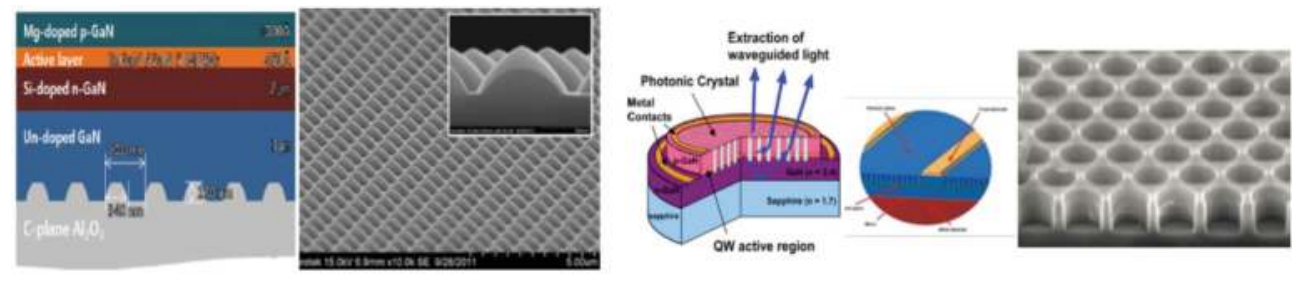

(a)

(b)

Figure 21. LED patterning using soft UV-NIL (a: NPSS-based LEDs; b: PhC LEDs) [19, 20]. 
promising applications for R2R thermal NIL is in the large-area fabrication of anti-reflective films, optical components and structures, particularly micro-lens structures.

LA-NIL has emerged as an effective approach to simultaneously control both the heterojunction morphology and polymer chains in organic photovoltaics. Currently, in the area of nanoimprinted polymer solar cells, much progress has been achieved in the fabrication of nanostructured morphology, control of molecular orientation/crystallinity, deposition of acceptor materials, patterned electrodes, understanding of structure-property correlations, and device performance [8]. Yang et al. reported low bandgap polymer solar cells with high efficiency of $5.5 \%$ can be achieved using NIL. This investigation indicates that solar cells made on the highest aspect ratio PCPDTBT nanostructures are among the best reported devices using the same material with a PCE of 5.5\%, and NIL fabrication works better for low bandgap polymer solar cells [42]. Highly efficient colored perovskite solar cells having $10.12,8.17$, and $7.72 \%$ of the PCE for the red, green, and blue (RGB) colors, respectively, have been demonstrated by Lee et al. [43]. In this study, the large-area Ag nanogratings were fabricated by NIL-based processes.

In recent years, a number of commercial applications have been discovered which require low cost, large-area patterning, particularly displays, optical coatings and films, and biological applications such as anti-fouling surfaces and micro-fluidic devices. Wire grid polarizers (WGPs) are able to provide effective polarizer with high light transmittance and high contrast ratio. They are also thermally stable and structurally simple making them ideal for use as integrated polarizer in ultraslim high performance display. Roller-type NIL has been utilized to mass produce the WGPSs on both the flexible and rigid substrates. A R2R UV-NIL process has been employed to fabricate the metal wire grid polarizers with nanoscale structures on a flexible substrate $[4-8,14-16]$.

\section{Prospects and challenges of LA-NIL}

Large-area NIL has been considered as a promising technology for cost-effective fabrication of sub-micron and nanopatterns over large areas. This technique has exhibited great potential and promising prospects in LEDs and HDD, and patterned substrates for full wafer imprinting, anti-reflective coatings or films with moth-eye structures, flexible electronics, and WGP for roll-to-roll imprinting, nanostructured glass for roll-to-plate type imprinting. Perhaps the application which is closest to mass production is anti-reflective coatings and films. LA-NIL has made great progress toward volume production.

Table 1 presented the summary and comparison of various large-area NIL processes. We can see that each process has its strength and weakness. According to the substrate type, imprint area, resolution, throughput, etc., it is necessary to determine the most suitable process for specific applications. Compared to the full wafer NIL, the roller-type NIL process possesses some distinctive advantages (lower imprint force and demolding force, better replication uniformity) because only a line area is in contact during imprinting. The line contact between the mold and the substrate during imprinting reduces the force for the complete filling, the effects of thickness unevenness and dust. Moreover, the roller-type NIL has more flexibility in the choice of replication geometry. In particularly, it has the unique capability of continuous patterning. R2R NIL process shows the highest yield rate. 


\begin{tabular}{|c|c|c|c|}
\hline \multirow[t]{3}{*}{ Item } & \multicolumn{3}{|c|}{ NIL process } \\
\hline & \multirow{2}{*}{$\begin{array}{l}\text { Full wafer NIL (plate-to-plate } \\
\text { type NIL) }\end{array}$} & \multicolumn{2}{|c|}{ Roller-type NIL } \\
\hline & & Roll-to-plate type NIL & Roll-to-roll type NIL \\
\hline Substrate & Rigid substrate & Rigid substrate & Flexible substrate \\
\hline Mold & $\begin{array}{l}\text { Flat molds (rigid, flexible, or } \\
\text { thin-film mold) }\end{array}$ & $\begin{array}{l}\text { Roller mold or belt-style mold } \\
\text { (flexible mold) }\end{array}$ & Roller mold (rigid or flexible mold) \\
\hline $\begin{array}{l}\text { Imprinting } \\
\text { area }\end{array}$ & $\begin{array}{l}\sim 300-400 \mathrm{~mm}^{2} \\
(450 \mathrm{~mm} \times 500 \mathrm{~mm})\end{array}$ & $\begin{array}{l}1 \mathrm{~m} \times 0.3 \mathrm{~m} \text { (extremely large- } \\
\text { area) }\end{array}$ & 300 mm (width) (13 inch) \\
\hline Resolution & Sub-20 nm & $40 \mathrm{~nm}$ & Sub-30 nm \\
\hline $\begin{array}{l}\text { Continuous } \\
\text { patterning }\end{array}$ & No & Yes & Yes \\
\hline Throughput & Moderate (350 disks/h) & High & Very high (Web 30 m/min) \\
\hline $\begin{array}{l}\text { Industrial } \\
\text { application }\end{array}$ & $\begin{array}{l}\text { LED, HDD, wafer-level optical } \\
\text { component }\end{array}$ & $\begin{array}{l}\text { Solar cell panel, architectural } \\
\text { windows, flat panel displays }\end{array}$ & $\begin{array}{l}\text { Anti-reflective film and coating, } \\
\text { flexible electronics, solar cells, } \\
\text { displays, resin mold, WGP }\end{array}$ \\
\hline $\begin{array}{l}\text { Representative } \\
\text { company or } \\
\text { institute }\end{array}$ & $\begin{array}{l}\text { SUSS, MII, Toshiba, Samsung, } \\
\text { ESCO, AIST }\end{array}$ & Hitachi, Rolith & $\begin{array}{l}\text { IMRE, EVG, Toshiba, HP, U } \\
\text { Michigan, U Mass, U Tokyo }\end{array}$ \\
\hline $\begin{array}{l}\text { Distinctive } \\
\text { features }\end{array}$ & $\begin{array}{l}\text { Surface contact, non-flat } \\
\text { substrate, peel demolding, gas- } \\
\text { assisted or roller press force }\end{array}$ & $\begin{array}{l}\text { Line contact, ultra-large-size } \\
\text { rigid substrate, belt-type mold, } \\
\text { extending cured time }\end{array}$ & $\begin{array}{l}\text { Line contact, high throughput, } \\
\text { large-scale production process, } \\
\text { roller mold }\end{array}$ \\
\hline $\begin{array}{l}\text { Challenging } \\
\text { issue }\end{array}$ & $\begin{array}{l}\text { Large-area demolding, large size } \\
\text { master fabrication }\end{array}$ & $\begin{array}{l}\text { Belt-type mold fabrication, } \\
\text { conformal contact }\end{array}$ & $\begin{array}{l}\text { Seamless roller mold fabrication, } \\
\text { coating method }\end{array}$ \\
\hline
\end{tabular}

Table 1. Summary and comparison of various large-area NIL processes.

Large-area imprinting technologies have made significant progresses. However, there are still many challenging issues, for example, defect, establishing an infrastructure, mold lifetime, large size master fabrication, uniformity of patterns and residual layer thickness across imprint field, discovering new applications and products especially suitable for large-area NIL, etc. Full wafer NIL is capable of wafer-scale processing at nanoscale resolution with relatively low cost. But the replica area is limited by the master mold size. It is difficult to ensure large-area conformal contact between the mold and substrate, especially for rigid stamp and non-flat substrate. For R2R UV-NIL process, it faces the following challenging issues: obtaining thin and uniform coatings, fabricating multi-layer nanostructures on flexible substrate, producing seamless cylindrical imprint molds and roller mold lifetime, functional imprinting materials with fast curing, low viscosity, and shrinkage. To address these challenges, an infrastructure of large-area imprinting should firstly be established. Figure 22 illustrated an infrastructure proposed by author. It mainly includes three aspects: the fundamental theory, the enabling technologies, and various industrial and potential applications. Practical demands form specific applications are main driving forces push large-area imprinting development and advances. The continuous and further research on fundamental theory and enabling technologies provide mass production capability for a variety of commercial applications. 
Applications: Users and Researchers

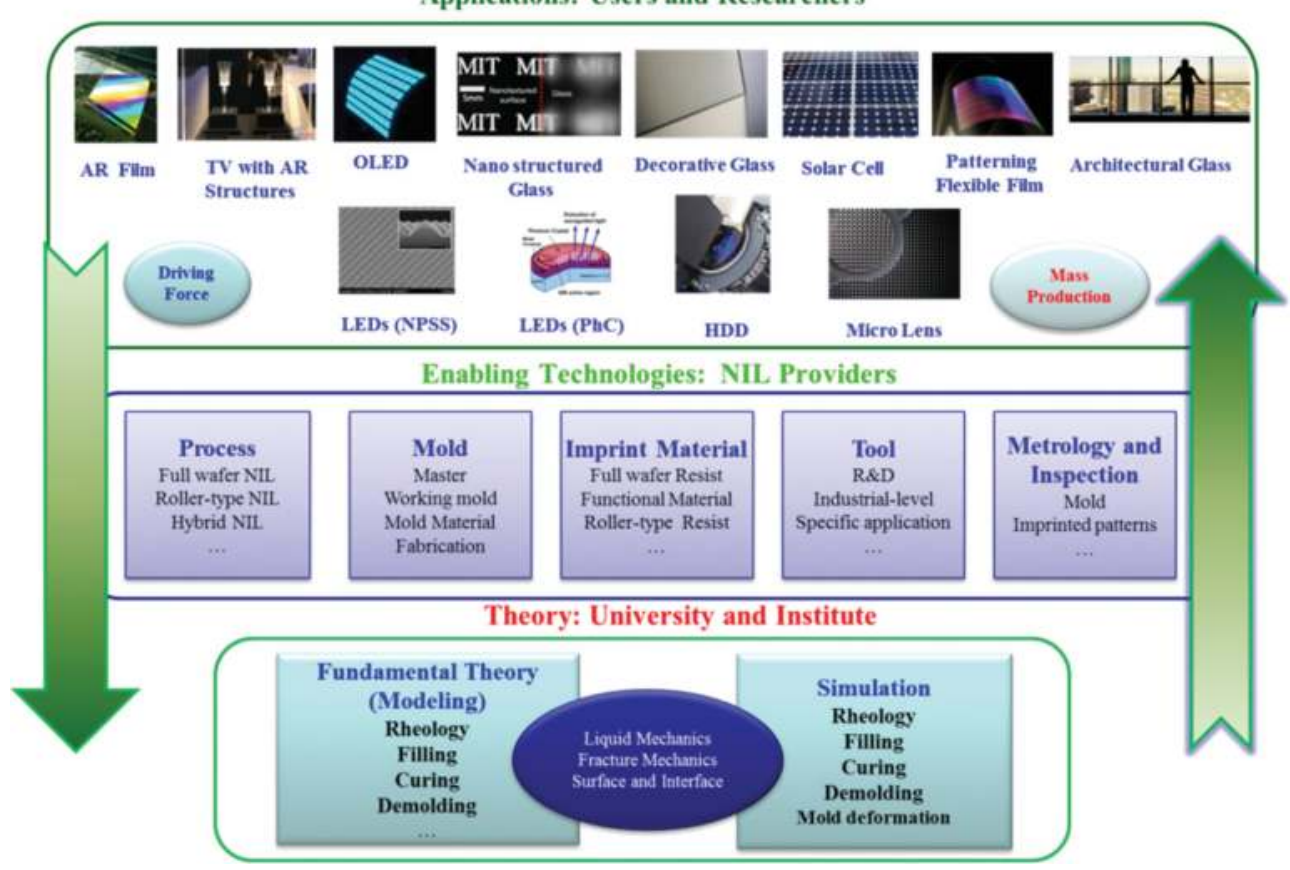

Figure 22. An infrastructure of large-area nanoimprinting.

Future trend for large-area imprinting process may be: film mold-based UV imprinting process, belt-type imprinting, R2R UV-NIL, fluoropolymer and PVA-based composite mold, large size seamless roller mold. Functional imprinting materials may be frequently used. Further enlarging imprinting area, improving resolution, and enhancing throughput and quality are needed.

\section{Conclusions}

NIL has over the two decade gone from being a new and exciting research topic, to be a technology used in the most advanced parts of various industries. Large-area NIL has been proven to be a cost-effective high volume nanopatterning method for large-area structure replication in the micrometer and nanometer scale, fabricating complex 3D micro/nanostructures, especially producing large-area patterns on the non-planar surfaces even curved substrates at low cost and with high throughput. In particular, it provides an ideal solution and a powerful tool for mass producing micro/nanostructures over large areas at low cost and high yield rate for the applications in HB-LEDs, anti-reflective coatings or films with moth-eye structures, flexible electronics, solar cells, architectural glass, optical elements, patterned media, micro-lens, OLED, wire grid polarizer, and functional polymer devices. That opens the way for many applications not previously conceptualized or economically feasible. 
Full wafer NIL is a promising large-area wafer-scale nanoimprint method with nanoscale resolution. R2R NIL is attracting interest by both academic and industry around the world because of its inherent advantages of low cost, high throughput, and large-area patterning. Future advances in R2R nanoimprinting and its utilization in commercial applications strongly depends on the development and fabrication of new roller mold and resist materials that can maintain the fidelity of the replicated features and deliver an almost defect-free pattern transfer for long process cycles in a high volume manufacturing process. Unlike the full wafer NIL, during the R2R NIL process the mold material undergoes a severe test of its reliability, durability, robustness, mold recoverability, and the ability to maintain the fidelity of pattern transfer throughout the continuous process. Therefore the roller mold is one of the most important components that can determine the outcome of the entire process [35].

R2R NIL has been regarded as the closest process for the industrial application of NIL. In particular, the applications in anti-reflective coatings or films, flexible electronics, and wire grid polarizer have demonstrated significantly commercial prospect. Large-area NIL will become more and more important for many applications in large-area patterning, fabricating 3D micro/nanostructures and creating patterns on the non-planar or curved surface. There is a plenty of room to enhance the resolution, patterning area, mold lifetime, and yield for the promising patterning method.

\section{Acknowledgements}

Parts of this chapter are taken from the authors' former work (Soft UV Nanoimprint Lithography and Its Applications, and Nanoimprint Lithography, published by INTECH). This work was financially supported by National Science Foundation of China (Grant Nos. 51375250 and 51775288), and Qingdao Innovative Talents Project (13-CX-18).

\section{Author details}

Hongbo $\operatorname{Lan}^{1,2 *}$

*Address all correspondence to: hblan99@126.com

1 Nanomanufacturing and Nano-Optoelectronics Lab, Qingdao University of Technology, Qingdao, China

2 Qindao Engineering Research Center for 3D Printing, Qingdao, China

\section{References}

[1] Verschuuren MA, Megens M, Ni Y, et al. Large area nanoimprint by substrate conformal imprint lithography (SCIL). Advanced Optical Technologies. 2017;6(3-4):243-264 
[2] Bläsi B, Tucher N, Höhn O, et al. Large area patterning using interference and nanoimprint lithography. Proceedings of SPIE. 2016;9888:98880H

[3] Schoot JV, Schift H. Next-generation lithography-An outlook on EUV projection and nanoimprint. Advanced Optical Technologies. 2017;6(3-4):159-162

[4] Lan H, Ding Y, Liu H. Nanoimprint Lithography Principles, Processes and Materials. New York, USA: Nova Science Pub Inc.; 2011

[5] Dumond JJ, Low HY. Recent developments and design challenges in continuous roller microand nanoimprinting. Journal of Vacuum Science and Technology B. 2012;30(1):010801

[6] Ok JG, Shin YJ, Park HJ, et al. A step toward next-generation nanoimprint lithography: Extending productivity and applicability. Applied Physics A. 2015;121:343-356

[7] Viheriälä J, Niemi T, Kontio J, et al. Nanoimprint lithography-Next generation nanopatterning methods for nanophotonics fabrication. In: Kim K, editor. Recent Optical and Photonic Technologies. Rijeka: InTech; 2010. pp. P275-P298

[8] Yang Y, Mielczarek K, Aryal M, et al. Nanoimprinted polymer solar cell. ACS Nano. 2012;6(4):2877-2892

[9] Lee YC, Tu SH. Improving the light-emitting efficiency of GaN LEDs using nanoimprint lithography. In: Cui B, editor. Recent Advances in Nanofabrication Techniques and Applications. Rijeka: InTech; 2011. pp. 173-195

[10] Baek JH, Kim SM, Lee IH, et al. Control of characteristic performance by patterned structure in light-emitting diodes. Proceedings of SPIE. 2011;7945:79451B

[11] Lan H, Liu H. UV-nanoimprint lithography: Structure, materials and fabrication of flexible molds. Journal of Nanoscience and Nanotechnology. 2013;13(5):3145-3172

[12] Chou Y, Krauss P, Renstrom P. Imprint lithography with 25-nanometer resolution. Science. 1996;272(5258):85-87

[13] Schift H. Nanoimprint lithography: An old story in modern times? A review. Journal of Vacuum Science and Technology B. 2008;26(2):458-480

[14] Guo J. Nanoimprint lithography: Methods and material requirements. Advanced Materials. 2007;19(4):495-513

[15] Kooy M, Mohamed K, Pin L, et al. A review of roll-to-roll nanoimprint lithography. Nanoscale Research Letters. 2014;9:320

[16] Ahn SH, Guo LJ. Large-area roll-to-roll and roll-to-plate nanoimprint lithography: A step toward high-throughput application of continuous nanoimprinting. ASC. Nano. 2009;3 (8):2304-2310

[17] Glinsner T, Plachetka U, Matthias T, et al. Soft UV-based nanoimprint lithography for large area imprinting applications. Proceedins of SPIE. 2007;6517:651718

[18] Verschuuren MA. Substrate conformal imprint lithography for nanophotonics [PhD thesis]. Utrecht University; 2011 
[19] Ji R, Hornung M, Verschuuren MA, et al. UV enhanced substrate conformal imprint lithography (UV-SCIL) technique for photonic crystals patterning in LED manufacturing. Microelectronic Engineering. 2010;87:963-967

[20] Shinohara H, Fujiwara S, Tashiro T, et al. Formation of patterned sapphire substrate using UV imprint processes. Journal of Photopolymer Science and Technology. 2013;26(1):113-117

[21] Sato Y, Mizawa T, Mizukami Y, et al. Development of film mold for roll to roll nanoimprintg process and its application. Journal of Photopolymer Science and Technology. 2013;26(1):125-128

[22] Youn S, Ogiwara M, Goto H, et al. Prototype development of a roller imprint system and its application to large area polymer replication for a microstructured optical device. Journal of Materials Processing Technology. 2008;202:76-85

[23] Roller Press Scan® method. http://www.engineeringsystem.co.jp/automation/nanoimprint/ $\mathrm{en} / .2017 / 9$

[24] Tan H, Gilbertson A, Chou S. Roller nanoimprint lithography. Journal of Vacuum Science and Technology B. 1998;16(6):3296-3298

[25] Ahn S, Cha J, Myung H, et al. Continuous ultraviolet roll nanoimprinting process for replicating large-scale nano- and micropatterns. Applied Physics Letters. 2006;89(21):213101

[26] Park S, Choi K, Kim G, et al. Nanoscale patterning with the double-layered soft cylindrical stamps by means of UV-nanoimprint lithography. Microelectronic Engineering. 2009;86(4-6): 604-607

[27] Nano-scale Pattern Productivity Enhanced 100 times by Sheet Nanoimprint Technology. Hitachi Research Laboratory. 2011

[28] Jiang W, Liu H, Ding $\mathrm{Y}$, et al. Investigation of ink transfer in a roller-reversal imprint process. Journal of Micromechanics and Microengineering. 2009;19:015033

[29] Frenkel R, Kim B, Yao D. Extrusion roller imprinting with a Variotherrm belt mold. Machines. 2014;2(4):299-311

[30] Nagato K, Sugimoto S, Hamaguchi $T$, et al. Iterative roller imprint of multilayered nanostructures. Microelectronic Engineering. 2010;87:1543-1545

[31] EVG®750R2R Automated Hot Embossing System. http://www.evgroup.com. 2017

[32] Ahn BS, Guo LJ. High-speed roll-to-roll nanoimprint lithography on flexible plastic substrates. Advanced Materials. 2008;20:2044-2049

[33] Jeans A, Almanza-Workman M, Cobene R, et al. Advances in roll-to-roll imprint lithography for display applications. Proceedings of SPIE. 2010;7637:763719

[34] Kim H, Almanza-Workman M, Garcia B, et al. Roll-to-roll manufacturing of electronics on flexible substrates using self-aligned imprint lithography (SAIL). Journal of the SID. 2009;17:963-970

[35] John J, Tang Y, Rothstein J, et al. Large-area, continuous roll-to-roll nanoimprinting with PFPE composite molds. Nanotechnology. 2013;24:505307 
[36] Inanami R, Ojima T, Matsuki K, et al. Sub-100 nm pattern formation by roll-to-roll nanoimprint. Proceeding of SPIE. 2012;8323:83231J

[37] Ok JG, Ahn SH, Kwak MK, et al. Continuous and high-throughput nanopatterning methodologies based on mechanical deformation. Journal of Materials Chemistry C. 2013;1:7681-7691

[38] Burghoorn M, Roosen-Melsen D, Riet J, et al. Single layer broadband anti-reflective coatings for plastic substrates produced by full wafer and roll-to-roll step-and-flash nano-imprint lithography. Materials. 2013;6:3710-3726

[39] Zhong ZW, Shan XC. Microstructure formation via roll-to-roll UV embossing using a flexible mould made from a laminated polymer-copper film. Journal of Micromechanics and Microengineering. 2012;22:085010

[40] Byeon KJ, Hong E, Park H, et al. Full wafer scale nanoimprint lithography for GaN-based light-emitting diodes. Thin Solid Films. 2011;519:2241-2246

[41] Lim H, Choi K, Kim G, et al. Roller nanoimprint lithography for flexible electronic devices of a sub-micron scale. Microelectronic Engineering. 2011;88:2017-2020

[42] Yang Y, Mielczarek K, Zakhidov A, et al. Efficient low bandgap polymer solar cell with ordered heterojunction defined by nanoimprint lithography. ACS Applied Materials \& Interfaces. 2014;6:19282-19287

[43] Lee KT, Jang JY, Zhang J, et al. Highly efficient colored perovskite solar cells integrated with ultrathin subwavelength plasmonic nanoresonators. Scientific Reports. 2017;7:10640 